\section{P79 Exploring the Dietary Behaviors and Perceptions of African Immigrants: A Qualitative Study of Nigerian and Congolese Immigrants}

Oluwatosin Akingbule, MS, CHES, University of Illinois Urbana-Champaign; Margarita Teran-Garcia, PhD, MD, FTOS, teranmd@illinois.edu, University of Illinois UrbanaChampaign, 2103 N Dunlap Ave, Champaign, IL, 61820; Olive Panumpabi, BS, University of Illinois UrbanaChampaign; Reginald Alston, PhD, University of Illinois Urbana-Champaign

Background: Studies examining the prevalence of cardiometabolic diseases in African immigrants show that they are healthier on arrival to the U.S., but develop worse risk profiles closer to that of the general population when they live 10 years or longer in the country. This change has been attributed to dietary acculturation.

Objective: To explore contextual factors associated with changes in dietary behaviors of recent immigrant families from Nigeria and Congo.

Study Design, Setting, Participants: Five online focus group interviews were conducted with recent Nigerian and Congolese immigrant adults $(n=20$; Female $=19$, Male $=1$ ). Three focus groups were conducted in English and 2 were conducted in French. Most of participants (70\%, $\mathrm{n}=14$ ) completed the voluntary demographics survey. On average, participants were 42 years old and had 4 children. Educationally, most had at least a bachelor's degree (72\%). Participants were recruited from churches with large population of African immigrants and through leaders of the immigrant communities from the Midwest. Participants discussed dietary practices for their families, including the challenges of maintaining a healthy diet after immigration to the U.S.

Measurable Outcome/Analysis: Preliminary findings from the ongoing thematic analysis are reported.

Results: Participants had high level of awareness of the influence of healthy food choices on risk factors for cardiometabolic diseases. The majority had the perception of "American food" as unhealthy, characterizing them as containing a high amount of sugar and salt. All participants preferred and mostly consumed foods they were familiar with before migration, including traditional African meals. Participants had several misconceptions regarding the nutritional value of certain food products. They reported that their school-age kids preferred Americanized over the traditional African meals. Participants and community leaders demonstrated high level of interest in receiving educational resources to make healthier food choices.

Conclusion: The findings will help to develop culturally tailored interventions to reduce diet-related risks associated with cardiometabolic diseases.

Funding: None.

\section{P80 Exposure to Healthy Food Increases Food Preferences Among Children and Youth in Low- Income School Settings}

Deepa Srivastava,PhD, dsr@ucanr.edu, University of California Agriculture \& Natural Resources, 4437-B South Laspina St., Tulare, CA, 93274

Objective: To increase healthy food preference through taste tests among children and youth

Use of Theory or Research: This study utilized the short-term outcome indicator of the SNAP-Ed evaluation framework to examine food preference intention at the individual level, from tasting a variety of foods from the 5 food groups, consistent with Dietary Guidelines recommendations.

Target Audience: K-8th graders in SNAP-Ed eligible schools across 2 counties of Central California.

Program Description: In 2019-20, Calfresh Healthy Living, UC (CFHL, UC) nutrition education program was implemented with tasting activities among K-8th graders. Youth taste tests occurred as part of the direct and indirect nutrition education, and policy, systems, and environment initiatives in collaboration with community partners.

Evaluation Methods: Nutrition educators administered the Youth Taste Testing Tool to capture student intention in food preferences immediately following food tastings. Descriptive analysis was performed. In Tulare county, 363 tastings were conducted and in Kings county, 106 tastings were conducted.

Results: In Tulare county, of the 7,614 students tasting variety of food, $14 \%$ of students tasted the target food before, $97 \%$ tried it today, $81 \%$ were willing to eat it again, and $79 \%$ were willing to ask for it at home. In Kings county, of the 1,920 students tasting variety of food, $31 \%$ of students tasted the target food before, $98 \%$ tried it today, $84 \%$ were willing to eat it again, and $82 \%$ were willing to ask for it at home. Foods tasted were from all food groups- fruits, vegetables, grains, proteins, and dairy and served raw, paired with a familiar food, cooked or included in a mixed dish.

Conclusion: Taste tests provide opportunity to try a variety of foods from 5 food groups that compose MyPlate which is a promising strategy for promoting healthy food preferences among children and youth. Healthy food preferences, in turn, are associated with healthy food consumption patterns.

Funding: Supplemental Nutrition Assistance Program Education. 